\title{
Incarcerated Morgagni hernia mimicking acute cholecystitis
}

\author{
Jeffrey Forris Beecham Chick • Nikunj Rashmikant Chauhan • \\ Jennifer H. Lai $\cdot$ Bharti Khurana
}

Received: 12 May 2012/ Accepted: 23 June 2012/Published online: 15 July 2012

(c) SIMI 2012

\section{Case report}

A 64-year-old man presented with sharp, constant right upper quadrant pain, nausea, and constipation for 2 days. The patient reported intermittent right upper quadrant pain for the prior 6 years, which was exacerbated by eating, but noted that over the past 2 days the pain had become more severe. The patient had previously undergone three right upper quadrant ultrasound studies that were all negative for acute cholecystitis. On presentation, examination was notable for focal tenderness to palpation over the xiphoid process. A right upper quadrant ultrasound study was completed, and demonstrated a single hepatic cyst and gallbladder sludge without evidence of gallstones, gallbladder wall thickening, pericholecystic fluid, or a sonographic Murphy's sign (Fig. 1). A chest radiograph was obtained, and demonstrated elevation of the right hemidiaphragm with colonic interposition in the right upper quadrant (Fig. 2). Computed tomography of the abdomen and pelvis with intravenous and oral contrast was performed, and demonstrated a large right-sided anterior diaphragmatic hernia containing distended loops of colon with short segment narrowing at the entrance as well as soft tissue stranding and simple fluid within the herniation sac, consistent with an incarcerated Morgagni hernia (Fig. 3). The patient underwent laparoscopic diaphragmatic hernia

J. F. B. Chick $(\bowtie) \cdot$ N. R. Chauhan · B. Khurana

Department of Radiology, Brigham and Women's Hospital,

Harvard Medical School, Boston, 75 Francis Street,

Boston, MA 02115, USA

e-mail: jchick@partners.org

J. H. Lai

Department of Internal Medicine, California Pacific Medical Center, San Francisco, CA 94115, USA repair with mesh, which was complicated by postoperative development of a right pneumothorax, but with eventual normalization of the chest radiograph (Fig. 4) and resolution of the right upper quadrant pain.

\section{Discussion}

Abdominal pain is the most common complaint, prompting upwards of $8 \%$ of all emergency department visits [1]. Right upper quadrant pain, in particular, is most often attributed to acute cholecystitis or hepatobiliary disease [1]. Unfortunately, upwards of one-third of patients initially suspected of having acute cholecystitis are ultimately found to have a different medical diagnosis, including cardiac, pulmonary, intestinal, and renal diseases [1].

Diaphragmatic hernias, although usually asymptomatic in the adult population, are a rare cause of right upper quadrant pain [2]. The Morgagni hernia, or retrosternal hernia, which was first described in 1769 by anatomist Giovanni Battista Morgagni, is the least common form of diaphragmatic hernia, and accounts for between 1 and $2 \%$ of all diaphragmatic hernias [2,3]. The Morgagni hernia, which may become strangulated or incarcerated, is characterized by herniation of the stomach, bowel, or liver through the foreman of Morgagni, which is located posterior to the xiphoid process of the sternum [2,3].

The majority of congenital diaphragmatic hernias are diagnosed prenatally during routine ultrasound studies $[4,5]$. In adults, however, the diagnosis of a diaphragmatic hernia is usually made incidentally. Posteroanterior and lateral radiographs may demonstrate a homogenous mass or gas filled bowel in the thorax with an associated diaphragmatic elevation [5]. Unfortunately, in some instances, such findings may be mistaken for right middle lobe 


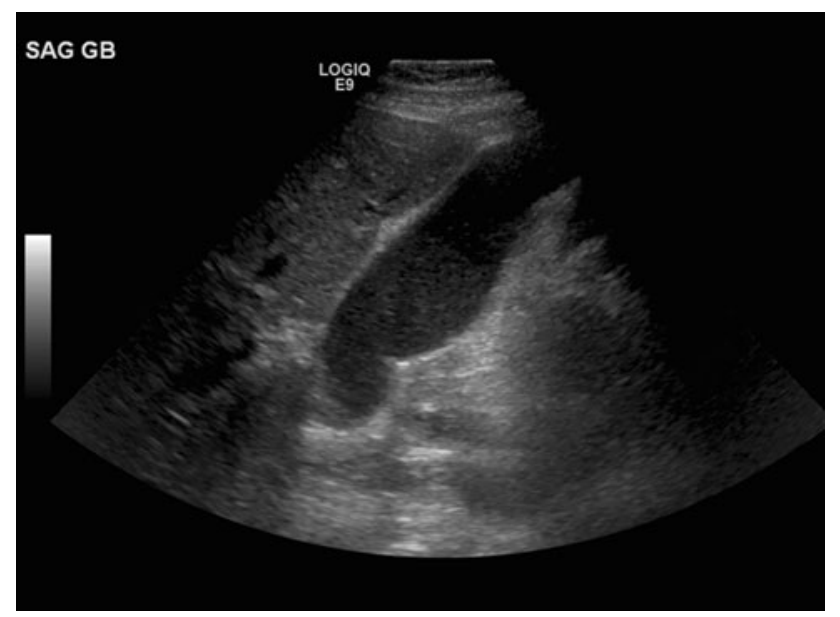

Fig. 1 Single gray scale image from a right upper quadrant ultrasound demonstrating gallbladder sludge, but no gallstones, gallbladder wall thickening, or pericholecystic fluid consolidation, a mediastinal mass, cardiomegaly, or a pericardial cyst [5]. Computed tomography, on the other hand, which is the imaging method of choice due to availability, speed, and reformatting capabilities, may demonstrate herniation of the stomach, liver, loops of bowel, or omentum into the chest through a retrosternal defect [5].

While the diagnosis of incarceration and strangulation cannot be made with imaging alone, such processes may be strongly suggested based on imaging characteristics. Incarceration, or an irreducible hernia, as demonstrated in this case, may be suggested when herniation occurs through a small defect, and the hernia sac has a narrow neck in association with bowel wall thickening, luminal dilatation, and surrounding soft tissue stranding with free fluid [5]. Early identification is of paramount importance as incarceration progresses to obstruction, inflammation,
Fig. 2 Frontal (a) and lateral (b) chest radiographs demonstrating elevation of the right hemidiaphragm with colonic interposition over the liver in the right upper quadrant
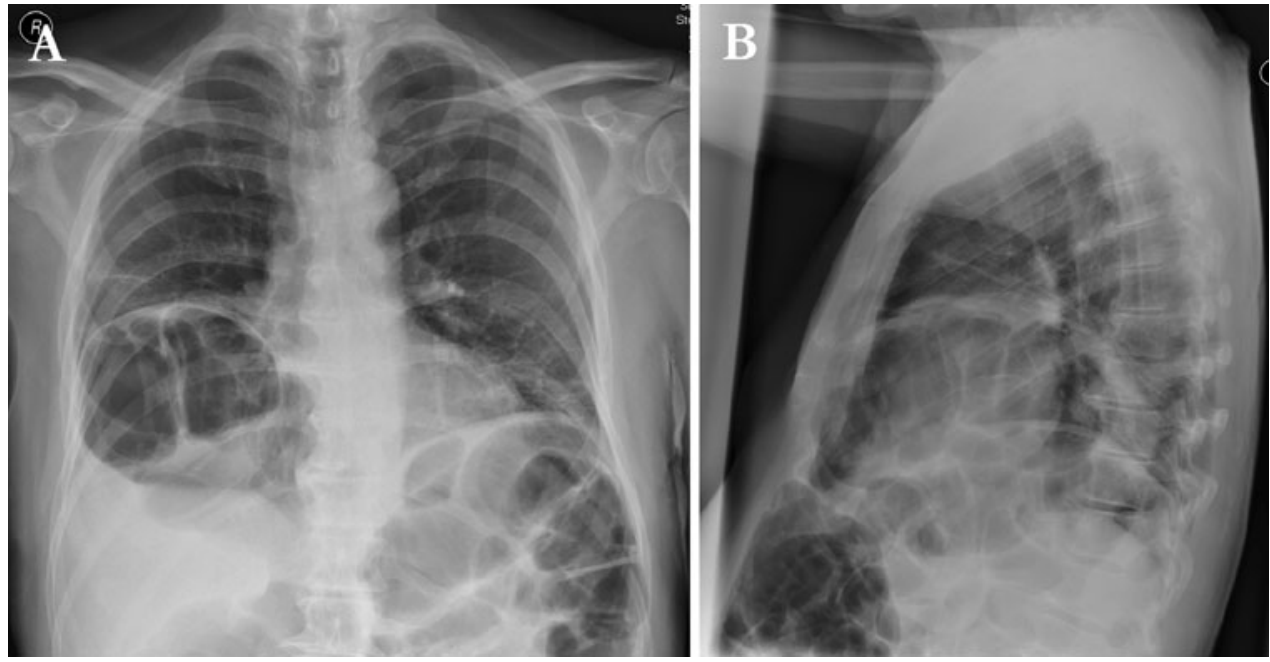
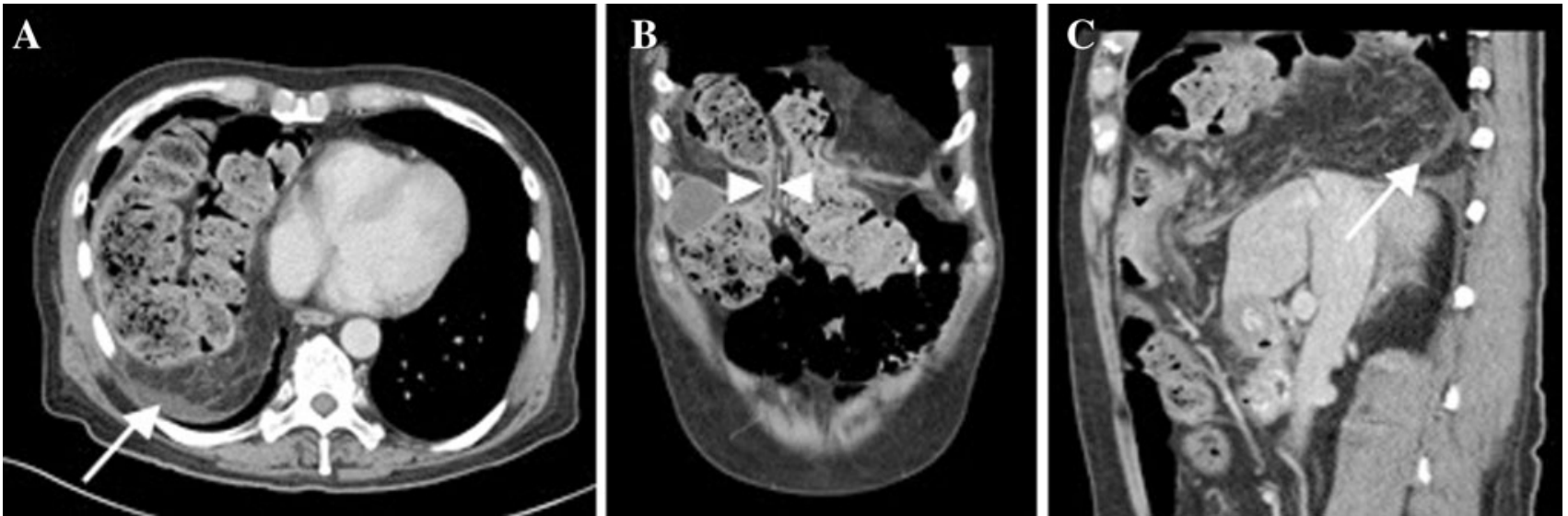

Fig. 3 Axial (a), coronal (b), and sagittal (c) images from computed tomography of the abdomen and pelvis with intravenous and oral contrast material demonstrating a large right anterior diaphragmatic hernia containing distended loops of hepatic flexure as well as narrowing of the colon as it enters the herniation sac (arrowheads) with soft tissue stranding and a moderate amount of fluid dependently (arrows), suggestive of an incarcerated Morgagni hernia 


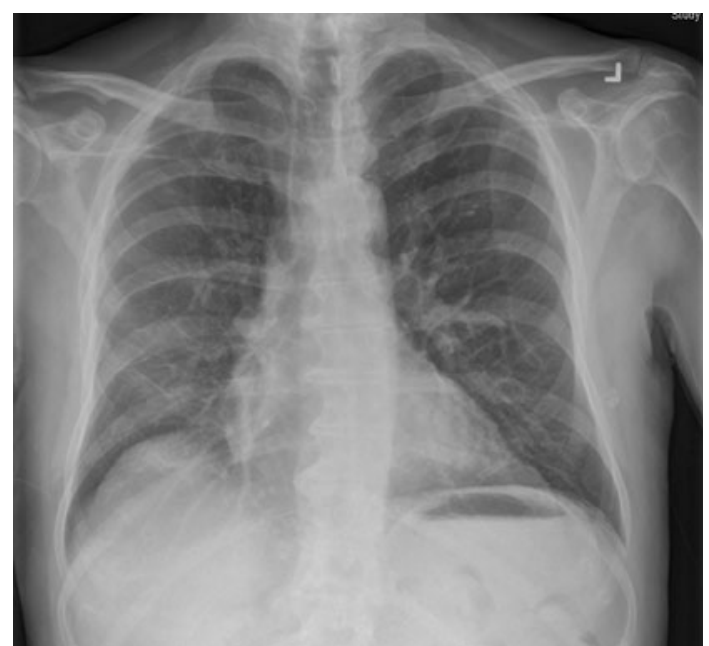

Fig. 4 Single frontal chest radiograph status post diaphragmatic hernia repair demonstrating resolution of previously seen elevated right hemidiaphragm

strangulation, ischemia, and bowel death [5]. In symptomatic patients, such hernias are surgically repaired as an emergency.
Conflict of interest None.

\section{References}

1. Laing FC, Federle MP, Jeffrey RB, Brown TW (1981) Ultrasonic evaluation of patients with acute right upper quadrant pain. Radiology 140:449-455

2. Morgagni GB (1769) The seats and causes of diseases investigated by anatomy, vol 3. Miller and Cadell, London, pp 205

3. Harrington SW (1951) Clinical manifestation and surgical treatment of congenital types of diaphragmatic hernia. Rev Gastroenterol 18:243

4. Graff LG, Robinson D (2001) Abdominal pain and emergency department evaluation. Emerg Med Clin North Am 19:123-136

5. Hanbidge A, Buckler PM, O'Malley ME, Wilson SR (2004) From the RSNA refresher courses: imaging evaluation for acute pain in the right upper quadrant. Radiographics 4:1117-1135 